\title{
An application of ICA to blind DS-CDMA detection: a joint optimization criterion
}

\author{
Iván Durán and Sergio Cruces ${ }^{\star}$ \\ Area de Teoría de la Señal y Comunicaciones, Escuela de Ingenieros, \\ Universidad de Sevilla, Camino de los descubrimientos s/n, \\ 41092-Sevilla, Spain \\ \{iduran, sergio\}@us.es
}

\begin{abstract}
This paper studies the application of the theory and algorithms of blind signal extraction to solve the problem of the detection of the desired users in a DS-CDMA communications system. Typically, the uplink in this system is characterized by users that transmit asynchronously and propagation channels that are multipath. We address inverse filter criterion introduced by Tugnait in [1] and we show that a prewhitening preprocessing approach together with the joint combination of several higher order statistics improves the detector performance.
\end{abstract}

\section{Introduction}

Direct sequence code division multiple access (DS-CDMA) is a technique widely extended in mobile communications $[3-5,1]$. In these systems users share the same band of frequencies and the same time slots, but they are separated in code. Each user transmits with a a different cyclic and quasi-orthogonal code (or sequence) that multiplies the user's symbols.

The main sources of errors at the detector are due to the multi-access interference, the inter-symbol interference, the asynchronism of users and the near-far problem. The multi-access interference (MAI) is a consequence of the fact that users transmit at the same time and in the same band of frequencies. The intersymbol interference (ISI) is due to the multi-path propagation in the channel and becomes important in systems with a high bit-rate since in this case the delays due to multi-path can spread up to the duration of one symbol [1]. The asynchronism of the users consist in that they start to transmit at a different time instants, while near-far problem is caused by the difference between the power transmission for the different users. These two last problems only occur at the up-link (from the mobile stations to the base stations), because in the down-link the base station transmits in a synchronous way and with the same power to all users [3].

Blind multiuser detection can be performed with the only knowledge of the desired users codes, avoiding the need for training sequences that lower the spectral efficiency [4].

* This work has been supported by the CICYT project of the Government of Spain (Grant TIC2001-0751-C04-04). 
In this work we pay special attention to the algorithm derived in [1] from an inverse filter criterion which maximizes the normalized fourth-order cumulant of the output. In this paper we show how the incorporation of prewhitening and the extension of the criterion to consider the joint optimization of several cumulant orders lead to a considerable improvement in the MSE of the detected user.

The paper is structured as follows. Section 2 presents the signal model while Sections 3 and 4.1 review the code constrained criterion and extraction algorithm introduced by [1]. In Section 4.2 we present our main contribution, the incorporation of prewhitening and the extension of the criterion to consider the joint optimization of several cumulant orders. In Section 5 we corroborate the theoretical behavior of the algorithms with simulations.

\section{System Model}

In the Blind Signal Extraction problem one typically considers the existence of $N$ independent source signals $\mathbf{s}(k)=\left[s_{1}(k), \ldots, s_{N}(k)\right]^{\mathrm{T}}$. These signals are combined, in presence of additive noise $\mathbf{n}(k)$, by a linear memoryless system characterized by the matrix $\mathbf{A}$, resulting the vector of $M$ observations

$$
\mathbf{x}(k)=\mathbf{A s}(k)+\mathbf{n}(k) .
$$

The recovery of the desired sources can be decomposed in two steps. The first step pre-whitens the observations, whereas the second step extracts the desired source. The prewhitened observations are

$$
\mathbf{z}(k)=\mathbf{W} \mathbf{x}(k)
$$

where $\mathbf{W}$ is the matrix which enforces the prewhitening $E\left[\mathbf{z}(k) \mathbf{z}(k)^{\mathrm{H}}\right]=\mathbf{I}_{M}$.

To obtain the desired source we multiply $\mathbf{z}(k)$ by a separation or extraction matrix $\mathbf{U}$ obtaining the output signal or estimated source

$$
y(k)=\mathbf{U z}(k)=\mathbf{G s}(k)+\mathbf{U W n}(k) .
$$

where $\mathbf{G}:=\mathbf{U W A}$ is the global transfer matrix from the sources to the output.

Next we will use the model of [1] to convert the detection of an user in a DSCDMA system into a problem of BSE with a linear and instantaneous mixture.

We will study a short-code DS-CDMA system (i.e., the spreading sequence has exactly one symbol of duration). In future high-capacity systems it will become more useful than long-code because it allows the multiuser receiver to know adaptively the MAI (multi-access interference) structure since the MAI in a symbol has identical statistics that the MAI in the next symbol [5].

We consider a system with $N_{u}$ users and $N_{c}$ chips per symbol. The $j$ th user's chip sequence is $\mathbf{c}_{j}=\left[\begin{array}{lll}c_{j}(0) & \cdots & c_{j}\left(N_{c}-1\right)\end{array}\right]^{\mathrm{T}}$. The symbol sequence transmitted by the $j$ th user is $\left\{b_{j}(k)\right\}$. The sequence $b_{j}(k)$ is zero-mean, independently and identically distributed (i.i.d.). For different $j \mathrm{~s}\left\{b_{j}(k)\right\}_{\mathrm{s}}$ are mutually independent. They are complex because the modulation can be quadrature or binary. 
The signal transmitted by the $j$ th user is therefore

$$
\hat{x}_{j}(n)=\sum_{k=-\infty}^{\infty} b_{j}(k) c_{j}\left(n-k N_{c}\right), \quad j=1,2, \ldots, N_{u} .
$$

This signal will pass through the corresponding linear and dispersive channel whose impulse response sampled at the chip interval $T_{c}$ is (for the $j$ th user) $g_{j}(n)$, which includes the effects of chip matched filtering at the receiver (see [5]) but not the transmission delay $\left(\bmod N_{c}\right)$ of $j$ th user, $d_{j},\left(0 \leq d_{j} \leq N_{c}-1\right)$. If we group the effect of the chip sequence and the channel in $h_{j}(n)$ we can write the contribution due to the $j$ th user at the receiver after to be sampled at $T_{c}$ as

$$
\begin{gathered}
\widetilde{x}_{j}(n)=\sum_{l=-\infty}^{\infty} b_{j}(l) h_{j}\left(n-d_{j}-l N_{c}\right) \\
h_{j}(n):=\sum_{m=0}^{N_{c}-1} c_{j}(m) g_{j}(n-m) .
\end{gathered}
$$

The total received signal $\widetilde{x}(n)$ is the superposition of contributions of the $N_{u}$ users plus an additive white Gaussian noise $w(n)$.

Now we will define the convolutional MIMO model (multiple inputs multiple outputs) from which we will build the instantaneous MIMO model to which apply the algorithms of blind extraction. If we define $\widetilde{\mathbf{x}}(k)=\left[\widetilde{x}\left(k N_{c}+N_{c}-\right.\right.$ 1) $\left.\cdots \widetilde{x}\left(k N_{c}\right)\right]^{\mathrm{T}}, \mathbf{h}_{j}(l)=\left[h_{j}\left(l N_{c}-d_{j}+N_{c}-1\right) \cdots h_{j}\left(l N_{c}-d_{j}\right)\right]^{\mathrm{T}}$ and $\widetilde{\mathbf{n}}(k)=$ $\left[w\left(k N_{c}+N_{c}-1\right) \cdots w\left(k N_{c}\right)\right]^{\mathrm{T}}$, the convolutional MIMO model is

$$
\widetilde{\mathbf{x}}(k)=\sum_{j=1}^{N_{u}} \sum_{l=0}^{L_{j}} \mathbf{h}_{j}(l) b_{j}(k-l)+\widetilde{\mathbf{n}}(k) .
$$

If we assume that multipaths delays are at most one symbol duration $\left(g_{j}(l)=\right.$ 0 for $l<0$ and $\left.l>N_{c}\right)$ and recalling that $0 \leq d_{j}<N_{c}$ we have $\mathbf{h}_{j}(l)=0$ for $l<0$ and $l \geq 3$. Thus $L_{j}=2$ for $j=1, \ldots, N_{u}$.

To convert the convolutional MIMO model in an instantaneous MIMO model we define $\mathbf{x}(k):=\left[\widetilde{\mathbf{x}}(k), \ldots, \widetilde{\mathbf{x}}\left(k-L_{e}+1\right)\right]^{\mathrm{T}}$ and $\mathbf{n}(k)$ in the same way, so that

$$
\mathbf{x}(k)=\mathbf{A} \mathbf{s}(k)+\mathbf{n}(k)
$$

being $\mathbf{s}(k)=\left[\widetilde{\mathbf{s}}(k), \ldots, \widetilde{\mathbf{s}}\left(k-L_{e}+1\right)\right]^{\mathrm{T}}$ the independent sources vector and

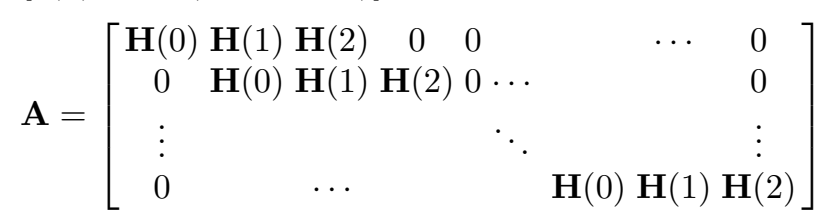

the matrix of linear and instantaneous mixture, being $\mathbf{H}(l)=\left[\mathbf{h}_{1}(l), \ldots, \mathbf{h}_{N_{u}}(l)\right]^{\mathrm{T}}$ and $\widetilde{\mathbf{s}}(k)=\left[b_{1}(k), \ldots, b_{N_{u}}(k)\right]^{\mathrm{T}}$.

The vector $\mathbf{x}(k)$ is $N_{c} L_{e} \times 1$, the matrix $\mathbf{A}$ is $N_{c} L_{e} \times N_{u}\left(L_{e}+2\right)$, and $\mathbf{s}$ is $N_{u}\left(L_{e}+2\right) \times 1$. Following the typical notation of BSS and BSE, $M=N_{c} L_{e}$ (number of observations) and $N=N_{u}\left(L_{e}+2\right)$ (number of independent sources). 


\section{Code constrained criterion}

We will address the code constrained criterion introduced by [1] to assure the extraction of the desired user. We will assume the use of the prewhitening process and will use an equivalent expression to obtain the projection matrix needed.

Let us assume we want to obtain the symbol sequence of the user $j_{0}$ with a delay $d$. Then, after prewhitening the observations given by (8), the extraction matrix $\mathbf{U}$ will be a row vector that in the absence of noise must satisfy

$$
\mathbf{U W A A}^{\mathrm{H}}=[0 \ldots \alpha \ldots 0] \mathbf{A}^{\mathrm{H}}=\alpha\left[\mathbf{h}_{j_{0}}^{\mathrm{H}}(d) \ldots \mathbf{h}_{j_{0}}^{\mathrm{H}}(0) 0 \ldots 0\right] .
$$

where $\alpha$ is a complex constant that goes at the position $j_{0}+N_{u} d$. Defining $\mathbf{h}_{j}^{(d)}:=\left[\mathbf{h}_{j}^{\mathrm{H}}(d) \ldots \mathbf{h}_{j}^{\mathrm{H}}(1) \mathbf{h}_{j}^{\mathrm{H}}(0)\right]^{H}$, and recalling that $g_{j}(l)$ is 0 for $l>N_{c}$ and for $l<0$, and $0 \leq d_{j}<N_{c}$, we have

$$
\mathbf{h}_{j}^{(d)}=\mathbf{C}_{j}^{(d)} \mathbf{g}_{j}
$$

being

$$
\mathbf{C}_{j}^{(d)}:=\left[\begin{array}{cccc}
0 & 0 & \cdots & 0 \\
\vdots & \vdots & \ddots & \vdots \\
c_{j}\left(N_{c}-1\right) & 0 & \cdots & 0 \\
c_{j}\left(N_{c}-2\right) & c_{j}\left(N_{c}-1\right) & \cdots & 0 \\
\vdots & \vdots & \cdots & \vdots \\
c_{j}(0) & c_{j}(1) & \cdots & 0 \\
0 & c_{j}(0) & \ddots & \vdots \\
\vdots & \vdots & \cdots & c_{j}\left(N_{c}-1\right) \\
0 & 0 & \ddots & \vdots \\
\vdots & \vdots & & c_{j}(0) \\
0 & 0 & & \\
\mathbf{g}_{j}:=\left[g_{j}\left(2 N_{c}-1-d_{j}\right)\right. & \cdots & \left.g_{j}\left(-d_{j}+1\right) g_{j}\left(-d_{j}\right)\right]^{\mathrm{T}} .
\end{array}\right.
$$

$\mathbf{C}_{j}^{(d)}$ is a Toeplitz $\left[(d+1) N_{c}\right] \times\left[2 N_{c}\right]$ matrix.

Now, using (10) and (11) and knowing that $\mathbf{A A}^{\mathrm{H}}=\mathbf{R}_{x x}=E\left\{\mathbf{x}(k) \mathbf{x}^{\mathrm{H}}(k)\right\}$ in the absence of noise, we can write

$$
\mathbf{R}_{x x} \mathbf{W}^{\mathrm{H}} \mathbf{U}^{\mathrm{H}}=\alpha \mathcal{C}_{j_{0}}^{(d)} \mathbf{g}_{j_{0}}
$$

where

$$
\mathcal{C}_{j_{0}}^{(d)}=\left[\begin{array}{c}
\mathbf{C}_{j_{0}}^{(d)} \\
\mathbf{0}
\end{array}\right]
$$

is a $N_{c} L_{e} \times 2 N_{c}$ matrix and $\mathbf{W}$ is the prewhitening matrix $\left(\mathbf{W R}_{x x} \mathbf{W}^{\mathrm{H}}=\mathbf{I}_{N_{c} L_{e}}\right)$.

Thus

$$
\mathbf{U}^{\mathrm{H}}=\alpha \mathbf{W} \mathcal{C}_{j_{0}}^{(d)} \mathbf{g}_{j_{0}}=\mathcal{C}_{j_{0}}^{1(d)} \alpha \mathbf{g}_{j_{0}}
$$


where $\mathcal{C}_{j_{0}}^{1(d)}:=\mathbf{W} \mathcal{C}_{j_{0}}^{(d)}$.

The projection matrix into the subspace of $\mathcal{C}_{j_{0}}^{1(d)}$ is

$$
\boldsymbol{\Pi}_{c}=\mathcal{C}_{j_{0}}^{1(d)}\left(\mathcal{C}_{j_{0}}^{1(d) \mathrm{H}} \mathcal{C}_{j_{0}}^{1(d)}\right)^{-1} \mathcal{C}_{j_{0}}^{1(d) \mathrm{H}}
$$

We have to post-multiply $\mathbf{U}$ by $\boldsymbol{\Pi}_{c}$ in the algorithms of separation or extraction to ensure that we obtain the desired user.

If we do not use prewhitening (like [1]) then $\mathcal{C}_{j_{0}}^{1(d)}:=\mathbf{R}_{x x}^{-1} \mathcal{C}_{j_{0}}^{(d)}$.

\section{Extraction algorithms}

In this section we begin presenting the existing implementation of the inverse filter criterion and we later consider the improvement of this algorithm with the joint optimization criterion.

\subsection{Algorithm derived from the Inverse Filter Criterion}

In [1] Tugnait et al. propose to use an inverse filter (equalizer) that apply on $\widetilde{\mathbf{x}}(k)$ to solve the extraction problem. This equalizer is adapted using a gradient algorithm that maximizes the criterion

$$
J_{42}(\mathbf{B})=\frac{\left|\operatorname{cum}_{4}(y(k))\right|}{\left(E\left\{|y(k)|^{2}\right\}\right)^{2}}
$$

which is based on the fourth-order cumulant $\operatorname{cum}_{4}(y(k)):=E\left\{|y(k)|^{4}\right\}$ $-2\left[E\left\{|y(k)|^{2}\right\}\right]^{2}-\left|E\left\{y(k)^{2}\right\}\right|^{2}$ of the output

$$
y(k)=\mathbf{B} \mathbf{x}=\sum_{i=0}^{L_{e}-1} \widetilde{\mathbf{B}}(i) \widetilde{\mathbf{x}}(k-i)
$$

where $\widetilde{\mathbf{B}}(i)$ is the equalizer of length $L_{e}$ symbols and $N \times 1$-dimensional.

In absence of noise, the recovery at the output of the desired $j_{0}$ th user up to a complex constant $\alpha \neq 0$, and an arbitrary delay $0 \leq d \leq L_{e}-1+L_{j_{0}}$

$$
y(k)=\alpha b_{j_{0}}(k-d)
$$

is blindly achieved through the maximization of (18) with respect to the row vector

$$
\mathbf{B}=\left[\widetilde{\mathbf{B}}(0), \widetilde{\mathbf{B}}(1), \cdots, \widetilde{\mathbf{B}}\left(L_{e}-1\right)\right]
$$




\subsection{Joint optimization criterion}

Assuming the prewhitening of the observations we have that $\mathbf{U}$ and $\mathbf{G}$ are row vectors both of unit 2-norm and the output takes the form $y(k)=\mathbf{U} \mathbf{z}(k)$.

We propose to estimate the desired independent component by jointly maximizing a weighted square sum of a combination of cumulants of the outputs with orders $r \geq 2$. A contrast function that achieves this objective is given by

$$
\psi_{\Omega}(y)=\sum_{r \in \Omega} \alpha_{r}\left|\operatorname{cum}_{r}(y(k))\right|^{2} \quad \text { subject to }\|\mathbf{U}\|_{2}=1 .
$$

where $\alpha_{r}$ are positive weighting terms. Let us define $q=\max \{r \in \Omega\}$, in our case, we choose to optimize the set of cumulant orders $\Omega=\{4,6\}$.

The problem with this approach is in the difficulty of the optimization of (22), which is highly non-linear with respect to $\mathbf{U}$. We can circumvent this difficulty by proposing a similar contrast function to (22) but whose dependence with respect to each of the extracting system candidates is quadratic, and thus, much more easy to optimize using algebraic methods.

Theorem 1. Considering a set of $q$ candidates for the extracting system $\left\{\mathbf{U}^{[1]}, \ldots, \mathbf{U}^{[q]}\right\}$ each of unit 2-norm, and the set of their respective outputs $\bar{y}=\left\{y^{[1]}, \ldots, y^{[q]}\right\}$, the following multivariate function

$$
\psi_{\Omega}(\bar{y})=\sum_{r \in \Omega} \frac{\alpha_{r}}{\left(\begin{array}{c}
q \\
r
\end{array}\right)} \sum_{\sigma \in \Gamma_{r}^{q}}\left|\operatorname{cum}\left(y^{\left[\sigma_{1}\right]}(k), \cdots, y^{\left[\sigma_{r}\right]}(k)\right)\right|^{2}
$$

where $\alpha_{r}>0$ and $\Gamma_{r}^{q}$ is the set of all the possible combinations $\left(\sigma_{1}, \cdots, \sigma_{r}\right)$ of the indexes $\{1, \ldots, q\}$ taken $r$ by $r$, is maximized at the extraction of one of the users, i.e., at this extreme point $y^{[1]}(k)=\cdots=y^{[q]}(k)=b_{j_{0}}(k-d)$.

A sketch for the proof of this theorem is presented in [2]. There is an interpretation of this theorem in terms of a low rank approximation of a set of cumulant tensors[6]. The contrast can be easily maximized optimizing $\psi_{\Omega}(\bar{y})$ cyclically with respect to each one of the elements $\mathbf{U}^{[m]}, m=1, \ldots, q$, while keeping fixed the others. In the following, it will be useful to refer to the variables that take a given value at the $i$-th iteration, this sequential notation will be denoted with the super-index ()$^{(i)}$. Due to the invariant property of $\psi_{\Omega}(\bar{y})$ with respect to permutations in its arguments, the cyclic maximization of the contrast with respect to $\mathbf{U}^{[m]}$ with $m=(i \bmod \mathrm{q})+1$, after assuming a projection step onto the symmetric subspace $y^{[1]}(k)=\cdots=y^{[q]}(k)$ that contains the solutions after each iteration, is equivalent to the sequential maximization of the function

$$
\begin{aligned}
\phi_{\Omega}\left(\mathbf{U}^{(i)}\right) & =\sum_{r \in \Omega} \frac{r}{q} \alpha_{r}\left|\operatorname{cum}_{r}\left(y^{(i)}(k), y^{(i-1)}(k), \ldots, y^{(i-1)}(k)\right)\right|^{2} \\
& =\mathbf{U}^{(i)} \mathbf{M}^{(i-1)} \mathbf{U}^{(i) H}
\end{aligned}
$$


w.r.t. the extraction system $\mathbf{U}^{(i)}$ through the iterations. Note that $\mathbf{M}^{(i-1)}$ is a constant matrix (as long as $\mathbf{U}^{(i-1)}, \cdots, \mathbf{U}^{(i-q+1)}$ are kept fixed) given by

$$
\mathbf{M}^{(i-1)}=\sum_{r \in \Omega} \frac{r}{q} \alpha_{r} \mathbf{c}_{\mathbf{z} y}^{(i-1)}(r)\left(\mathbf{c}_{\mathbf{z} y}^{(i-1)}(r)\right)^{H}
$$

where $\mathbf{c}_{\mathbf{z} y}^{(i-1)}(r)$ may be defined as

$$
\mathbf{c}_{\mathbf{z} y_{i}}^{(k-1)}(r)=\operatorname{Cum}\left(\mathbf{z}(k), y^{(i-1)}(k), \cdots, y^{(i-1)}(k)\right) .
$$

At each iteration the maximization $\phi_{\Omega}\left(\mathbf{U}^{(i)}\right)$ is obtained finding the eigenvector associated to the dominant eigenvalue of $\mathbf{M}^{(i-1)}$. Starting from the previous solution, if one considers to use $L$ iterations of the power method to approximate the dominant eigenvector (in practice $L=1$ or 2 works well), the following extraction algorithm is obtained

$$
\begin{aligned}
& \underline{\mathbf{U}}^{(0)}=\mathbf{U}^{(i-1)} \\
& \text { FOR } \quad l=1: L \\
& \quad \underline{\mathbf{U}}^{(l)}=\frac{\sum_{r \in \Omega} \frac{r}{q} \alpha_{r} d_{y}^{(l-1)}(r)\left(\mathbf{c}_{\mathbf{z} y}^{(i-1)}(r)\right)^{H}}{\left\|\sum_{r \in \Omega} \frac{r}{q} \alpha_{r} d_{y}^{(l-1)}(r)\left(\mathbf{c}_{\mathbf{z} y}^{(i-1)}(r)\right)^{H}\right\|_{2}} \\
& \begin{array}{l}
\mathrm{END} \\
\mathbf{U}^{(i)}=\underline{\mathbf{U}}^{(L)}
\end{array}
\end{aligned}
$$

where $d_{y}^{(l-1)}(r)=\underline{\mathbf{U}}^{(l-1)} \mathbf{c}_{z y}^{(i-1)}(r)$.

\section{Simulations}

In this section the comparison of the algorithms is done in terms of the mean square error criterion between the output and the symbol sequence of the desired user.

We made two different simulations: one in which all users are received with the same power and one in which the difference between the power of the desired user and one of the other users is $10 \mathrm{~dB}$ (near-far situation).

In each simulation we consider an observations time of 200 symbols and three 4-QPSK users with 8 chip/symbol. The channel was formed by four random multi-paths and the equalizer length was set to $L_{e}=3$.

We compared the results of the algorithm of [1] with and without prewhitening, and the algorithm of combined cumulants with $q=6$ and $\alpha_{4}=\alpha_{6}=0.5$.

In Fig. 1 we show the results of the simulations. We can see that the prewhitening reduces the MSE between the output and the desired user and that the reduction is higher if we use the proposed algorithm.

We should note that occasionally, for very low SNR, initialization process may fail when prewhitening is considered. When this happens the algorithm fail to obtain the desired user. This could be avoided if the system is conveniently reinitialized whenever this problem is detected. 

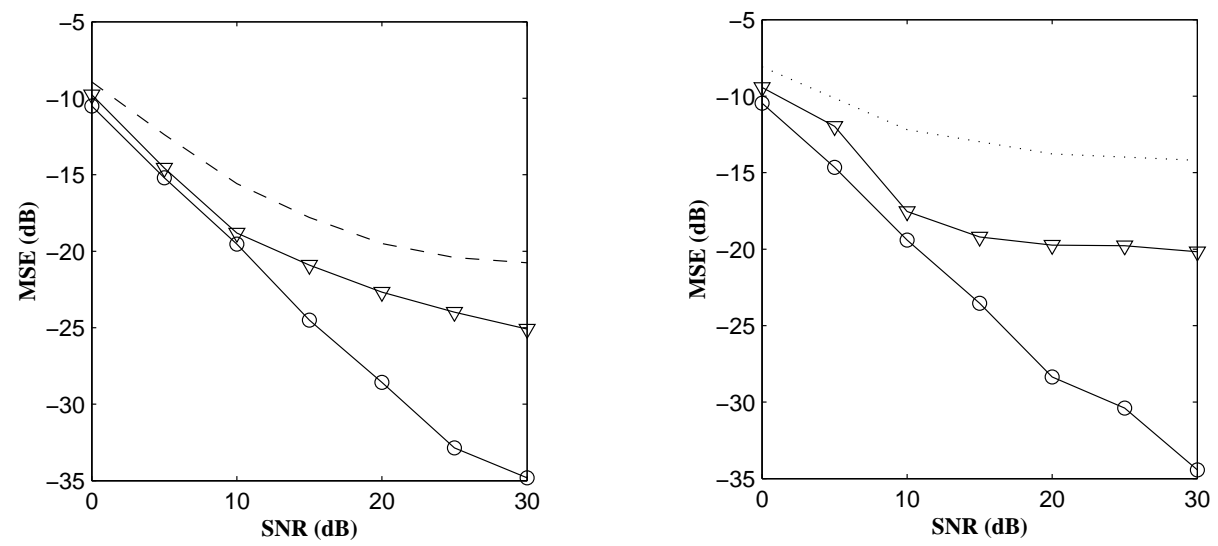

Fig. 1. Figures show the MSE between the output and the desired user over 100 Monte Carlo runs: ' $\triangle$ ' and dash line denote the algorithm (18) with and without prewhitening, respectively, whereas 'o' denotes the proposed algorithm. The MAI was 0dB for the simulation in left figure and $10 \mathrm{~dB}$ for that shown in the right figure.

\section{Conclusions}

In this paper we have addressed the problem of the blind extraction of a desired user in a DS-CDMA communications system. We show how to extend the inverse filter criteria with code constrained presented in [1] to consider a more general criterion based on joint optimization of several higher order statistics. The simulations corroborate how this later criterion improves MSE between the extracted symbol sequence and that of the desired user.

\section{References}

1. Tugnait, Jitendra K. and Li, Tongtong: Blind Detection of Asynchronous CDMA Signals in Multipath Channels Using Code-Constrained Inverse Filter Criterion. IEEE Trans. Signal Processing, Vol. 49, No. 7, pp. 1300-1309, Jul. 2001

2. Cruces S., A. Cichocki, A.: Combining Blind Source Extraction with Joint Approximate Diagonalization: Thin Algorithms for ICA, Accepted in the 4rd international conference on Independent Component Analysis and Blind Signal Separation. Nara, Japan. April, 2003.

3. Schniter, Phil and Johnson Jr., C. Richard: Minimum-Entropy Blind Acquisition/Equalization for Uplink DS-CDMA. Allerton Conference on Communication, Control and Computing, 1998.

4. Gesbert, D., Sorelius, J. and Paulraj, A.: Blind Multiuser MMSE Detection of CDMA Signals, Proc. of ICASSP, 1998.

5. Madhow, Upamanyu: Blind Adaptive Interference Suppression for Direct-Sequence CDMA. Proceedings of the IEEE, Vol. 86, No. 10, pp. 2049-2069, Oct. 1998

6. De Lathauwer, L., Comon, P., De-Moor, B., and Vandewalle, J.: Higher-order power method - application in independent component analysis. International Symposium on Nonlinear Theory and Applications NOLTA, Dec. 1995. 